\title{
The Product Collaborative Design System Architecture Based on Cloud Manufacturing Services
}

\author{
Dongfang $\mathrm{Hu}^{\mathrm{a}^{*}}$ and Ruonan Lei ${ }^{\mathrm{b}}$
}

\author{
School of Mechantronics Engineering, Henan University of Science and Technology, Luoyang, \\ 471003, China \\ a hdf@haust.edu.cn, b leiruonan1992@163.com
}

\begin{abstract}
Keywords: Cloud Manufacturing; Cloud Manufacturing Service; Product Collaborative Design; Functional Requirements
\end{abstract}

\begin{abstract}
In order to further improve the efficiency of product collaborative design method. Through deep research on product collaborative design and cloud manufacturing services, the method of applying cloud manufacturing service technology in the field of product collaborative design is put forward. Based on the analysis of cloud computing service model, the cloud computing service model is introduced into the current demand of manufacturing services, and the functional requirements of collaborative design system based on cloud manufacturing services are put forward. Through the design of a five layer system architecture, including resource layer, cloud layer, cloud service layer, application layer and user layer from the bottom to the top, the collaborative design system architecture based on cloud manufacturing services is established.
\end{abstract}

\section{Introduction}

Cloud manufacturing is a new manufacturing model, which develops based on networked manufacturing $^{[1]}$, grid manufacturing ${ }^{[2]}$, cloud computing ${ }^{[3]}$ and other technologies. Cloud manufacturing. The purpose of cloud manufacturing is to integrate all kinds of manufacturing resources, to build a huge resource cloud pool in the support of virtualization technology. So that the resources can be shared and utilized efficiently, and the on-demand manufacturing services can be provided to the user $^{[4]}$. As a new manufacturing model, the concept of cloud manufacturing has been widely studied since it was put forward in 2010. For the concept of cloud manufacturing, many experts and scholars at home and abroad have their own unique understanding of the way ${ }^{[5,6]}$, but the efficient sharing of resources and the use of the service is the consensus of all agree. Cloud manufacturing architecture is the basis for the implementation of cloud manufacturing concepts, and the cloud manufacturing architecture has the characteristics of high openness, which provides a great convenience for the application of the cloud manufacturing architecture in the related fields. Cloud manufacturing technology and networked collaborative design has become an important research field of manufacturing information in the world. Among them, the theoretical research on the construction and management of service environment and the problem of resource sharing are the focuses, but the key technologies of cloud manufacturing resource encapsulation and access control are lack of in-depth research $^{[7]}$. At the same time, there are few practical applications of cloud manufacturing technology, and the application of cloud manufacturing technology in the field of product collaborative design is rarely reported. Therefore, it is necessary to in-depth research the application of cloud manufacturing service in product collaborative design.

\section{The Requirements of Product Collaborative Design System Based on Cloud Manufacturing Services}

Now, how to improve the design efficiency of complex products, ensure the design quality of the products, reduce the design cost of the products, and meet the diversified demand of the customers are the important factors of enterprise survival and development[8]. Product design is a key link in the process of product quality and manufacturing cost, making full use of existing manufacturing resources, 
and making it play to the largest role are the optimal strategy of reducing cost of product design. Advanced equipment, senior experts, design knowledge and information are required precious resources for product manufacturing. At present, many enterprises exists a phenomenon of low resource utilization rate, which causes the waste of resources. Therefore, how to make these resources for effective organization and management, and improve the utilization rate of them will be the focus of the product collaborative design research.

Although traditional system of distributed network builds environment that can cooperative work, its limited hardware and software system of static, and not satisfactory with meeting the needs of the current collaborative design on system dynamics. Fundamental characteristic of cloud manufacturing service system can share existing resources sufficiently and decrease the cost of product design, which become the urgent requirement of modern product collaborative design.

In view of the above analysis, in this paper, the product collaborative design system of cloud manufacturing services mainly includes six fields that collaborative design requirements, user personalized requirements, design data sharing requirements, dynamic openness requirements, full life cycle service requirements and system interactive methods requirements. As shown in fig.1.

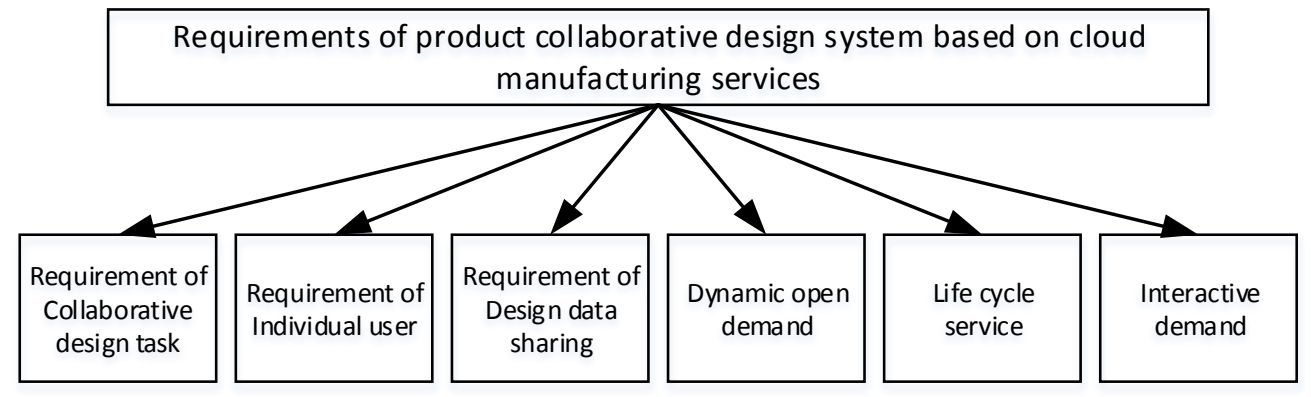

Figure 1. Cloud manufacturing services products collaborative design system requirements

1) Requirements for collaborative design tasks. The complexity of modern product design is getting higher and higher. In order to reduce the complexity of task difficulty and accelerate the execution speed of design tasks, the design task is usually decomposed into several subtasks. Then these subtasks are assigned to different design groups. This requires frequent communication and collaboration between design groups.

2) Demands of individuation. With the development of science and technology and the emergence of new market competition, people are increasing the requirements of all aspects of the product. The products in the design process in addition to considering the function, quality and price of these general factors, but also should consider the factors of product appearance, development speed etc.

3) Requirements for design data sharing. Data sharing in design process is the key to realize collaborative design. In the process of product collaborative design in cloud manufacturing service environment, it will involve a great deal of knowledge data, design data and simulation data etc. These data will change dynamically with the design task.

4) Requirements for dynamic opening. In the process of product collaborative design in cloud manufacturing service environment, executive organization of collaborative design tasks is random formation. Members need to be highly integrated and transparent to each other in executing the task.

5) Requirements for the whole life cycle of service. At the beginning of the product design, we must take into account the needs of the product, design, manufacture, use, maintenance, recycling and other products of all stages of the life cycle, and formulate the corresponding strategy to constrain. In order to avoid and reduce rework caused by design errors during the design phase, as far as possible to ensure that the product in the later stage of design can control all the relevant factors in the life cycle. Strive to achieve a one-time success of product design. 


\section{The Architecture of Collaborative Design System Based on Cloud Manufacturing Services}

Cloud manufacturing architecture as a basic manufacturing service framework can solve the manufacturing resource sharing, resource management, security management and other issues based on manufacturing grid, cloud computing, internet of things and other technologies. And it can be used in the dynamic, heterogeneous environment, multi design teams to interact with each other and share resources efficiently ${ }^{[9,10]}$. The system architecture of product collaborative design which is proposed based on cloud manufacturing service is the extension and application of cloud manufacturing architecture in product collaborative design field. The purpose of architecture is to realize the on-demand service and efficient sharing of the resources needed in the product collaborative design, reduce waste of resources and improve the utilization of design resources. The product collaborative design system architecture based on cloud manufacturing services is divided into five layers from bottom to top, as shown in Fig. 2.

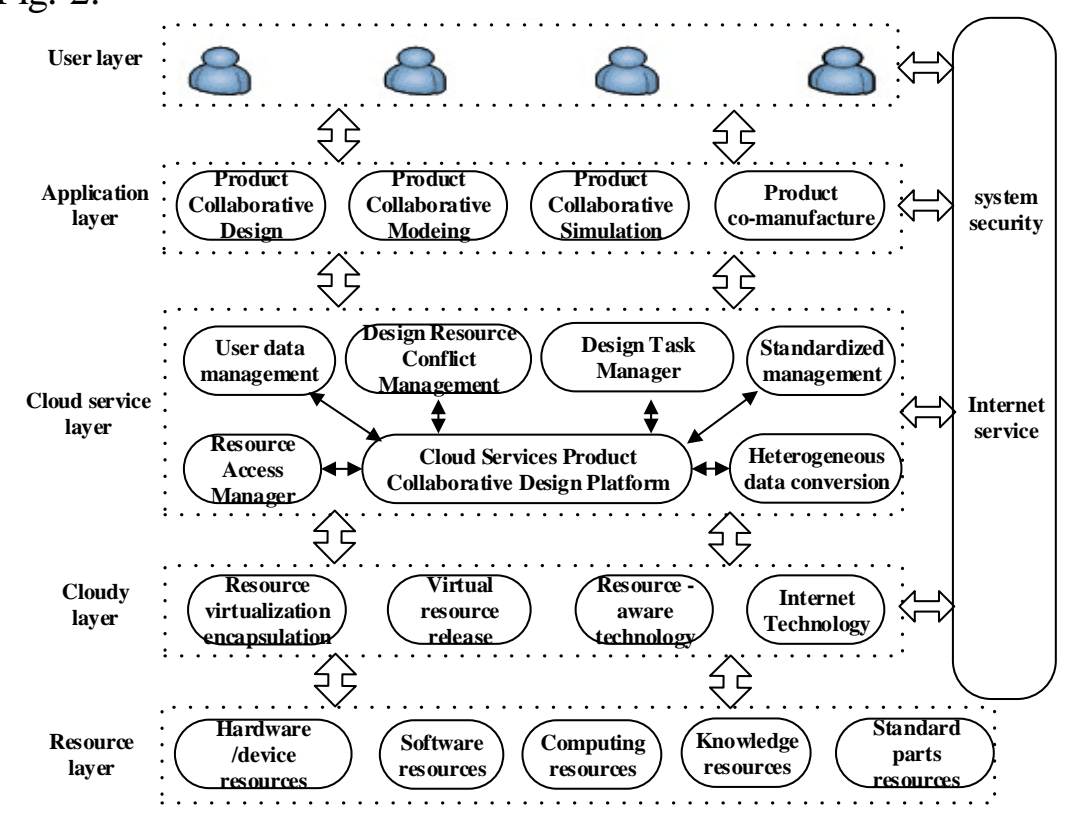

Figure 2. Collaborative design system architecture based on cloud manufacturing services

Resource Layer. The resource layer is the physical basis of product collaborative design. Design resource is a collection of resources in the product collaborative design system of cloud manufacturing services, such as hardware equipment, software, computing power, knowledge, standard parts, etc. Cloud manufacturing service environment is very rich in resources, it is necessary for designers to select design resources which is useful for their design tasks. In order to evaluate and control the implementation of collaborative design process effectively, designers need to have certain network knowledge to understand the topology and layout of design resources. For the vast number of resources in the cloud manufacturing service environment, designers do not have to take account of the actual location and layout of design resources, just need to call on-demand. The system can allocate related design resources dynamically and intelligently, which will reduce the network technical requirements of designers and the phenomenon of resources overload or idle, and improve the utilization of resources greatly.

Cloud Layer. In the collaborative design system based on cloud manufacturing services, the key to realize the product collaborative design is to solve the problem of design resource sharing in cloud manufacturing service environment. Through cross regional and enterprise to share design resources, which can avoid duplication of investment in the process of the design resources. And it improve resource utilization and reduce product design costs. The design resources in the product collaborative design system of cloud manufacturing services are characterized by diversity, heterogeneity and specificity. In order to realize the efficient sharing and cooperation of these resources, the first thing to be solved is to describe and encapsulate design resources. The main goal of the cloud layer is to shield 
the heterogeneity and complexity of these design resources, access to cloud manufacturing service platform, and provide a unified service call interface.

Resource packaging is an important part of the implementation of the cloud layer. For the package of software and knowledge resources, the WSDL can be described by XML language, and the corresponding service function interface can be extracted. Then, it can be accessed to the cloud manufacturing services in the product collaborative design system for the release of services by the network, users can call the relevant services on demand. Because of large size and poor working environment of hardware resources, we can realize the virtualization of resources by using the technology of resource aware and internet of things.

Cloud Service Layer. The cloud service layer is the key to the operation of the cloud co-design service system. In the cloud service layer, mainly including user management, standardized management, design task allocation, heterogeneous data conversion, design resource access conflict resolution and system security access control.

1) User Management

User management refers to the process of collaborative design in the product, according to the task needs of the implementation, the system can add and delete the designer intelligently, and record the execution history of the designer to ensure the task efficiency and orderly implementation.

2) Standardized management

Standardization management is the premise to provide efficient service, through the formulation of uniform standards for different resources, resource registration and call can be realized, the response time of design resource services will be reduced. And through the formulation of the corresponding service quality evaluation criteria, changes in the quality of service are monitored, the failed service is cleared in time, so that the system will provide high quality design services.

3) Design assignments

Task allocation is the key link of product collaborative design. The collaborative design system of cloud manufacturing service first decomposes the task in an intelligent way, and then allocates its corresponding tasks based on the ability of service resources.

4) Heterogeneous data conversion

In the process of collaborative design, due to the different habits of designers, the software systems used are not the same, then a large number of heterogeneous data will be produced. So how to realize the sharing of heterogeneous data becomes a bottleneck in the development of collaborative design. However, through the establishment of heterogeneous data conversion mechanism can effectively solve this problem, such as STEP / XML conversion mechanism, which can achieve the conversion through the establishment of STEP and XML data language mapping relationship.

5) Design resource access conflict

Product collaborative design is the process of conflict generation and digestion. For the same design resources, different users to access it, then it will be inevitably conflict in time. So how to effectively eliminate the conflict to ensure the efficiency of resource access is still an important issue, which need to be solved for the core service layer.

6) System security

The security of the system is the prerequisite to ensure the normal operation of the system, including the security of the user information, the safety of the design data and the security of the access process.

Application Layer. The application layer is the most intuitive performance layer of the product design system in the cloud manufacturing service. The functions are realized by establishing the corresponding service function interface, including product collaborative design, collaborative modeling, collaborative simulation, collaborative manufacturing and aftermarket management wait. Users can also conduct functional combination and reconstruction to meet the needs of collaborative design tasks at the maximum.

User Layer. The user layer is also the portal layer of the system. The user layer provides the user with a working environment which is independent of the operating system based on the network. Users can perform tasks such as resource access, service invocation, task submission and task monitoring in this 
environment. In addition, users can also query the history of resources and other information services, and select the inexpensive resources for their own use to minimize the cost of use. In recent years, with the development of computer technology, the user demand for the friendly interface of the system is also more and more high, so a good interactive interface is very important for cloud manufacturing services product design system.

\section{Summary}

In view of the current manufacturing requirements, the architecture and service model of cloud computing are studied. The requirements of product collaborative design system based on cloud manufacturing services are analyzed from six aspects. Then, the extension and application of cloud manufacturing architecture in the field of product collaborative design are studied. Finally, system architecture of the product collaborative design based on cloud manufacturing services is obtained, which realize the on-demand service and efficient sharing of the resources needed in the product collaborative design, and reduce waste of resources and improve the utilization of design resources.

\section{Acknowledgements}

The authors gratefully acknowledge the National Nature Science Foundation (No. 51475146).

\section{References}

[1] Z.H Liu, K Yan: Advanced Materials Research, Vol. 945-949 (2014) No.2, p.3450-3453.

[2] J.Z Fu: International Journal of Advanced Manufacturing Technology, Vol. 74 (2014) No.1-4, p.335-340.

[3] N. Farrukh, Q. Rizwan: Journal of Computer Science and Technology, Vol. 30 (2015) No.3, p.639-654.

[4] X.X Meng, S.J Liu and L Wu: Journal of Shandong University of Technology, Vol. 41 (2011) No.5, p.13-21 (In Chinese).

[5] L Ren, J Cui and N Li: Journal of Manufacturing Science and Engineering, Vol. 137 (2015) No.4.

[6] T Wang, S Guo and C G Lee: The International Journal of Advanced Manufacturing Technology, Vol. 71 (2014) No.9, p.2017-2031.

[7] W Fu: Research on several key technologies of cloud manufacturing and its preliminary development of prototype system (Ph.D., Zhejiang University, China 2012).

[8] C Yin, B.Q Huang and F Liu: Computer Integrated Manufacturing Systems, Vol. 17 (2011) No.3, p.496-503 (In Chinese).

[9] Q Li, B Qin and F Bai: Forging \& Stamping Technology, Vol. 36 (2011) No.3, p.140-143 (In Chinese).

[10] Q Zhang, C Qi: Journal of South China University of Technology (Natural Science Edition), Vol. 39 (2011) No.12, p.75-80 (In Chinese). 